\title{
Low Cost Module for Magic Glove using Robot Car
}

\author{
Raed S. M. Daraghma \\ Department of Electrical and Electronics Engineering, Palestine Technical University, Palestine \\ R.daraghmeh@ptuk.edu.ps
}

\begin{abstract}
The automation means employing of different automatic control for utilizing any device. The main object for many applications of the automation is to minimize human intrusion. In this paper, we design a new application for a glove controlled car using Wi-Fi technique via mobile systems. The new design enhances the extending range of ordinary Radio Frequency car employing Wi-Fi technique and reducing the overshoot phenomenon. We can conclude that from our design, the performance of the controlled car relies on the equipment signal strength, we extend the maximum range line of sight more than $300 \mathrm{~m}$ outdoors by using oriented external antennas in all directions. also, we propose a car controlling by hand glove, that translates the gesture of the hand fingers using flex sensor wirelessly to the Wi-Fi module that in turn transmits it to the car, the car translates the signal that the flex sensor and accelerometer sensor sent, then it will convert to motion and speed.
\end{abstract}

Keyword - Wi Fi, Arduino Uno, Flex sensor, DC motor.

\section{INTRODUCTION}

Robotic is the section of technology that transacts with styling. These technologies transact with automated machines that occupy the site of human.

The main interest in designing of robot is to monitor it smoothly and properly. There are various techniques to implement the work.

In paper [1], the author developed a spying device that knows the location and activities of user without his knowledge.

In paper [2], the author designed a new robot, he used cameras and sensors, these devices act as interface to monitor the robot with some manipulators, the author used other patterns such as: movements of hands, colour tracking, face recognition, finger tracking and some templates.

In paper [3], the author developed a new robot, this robot used in ball tracking and football games. The main disadvantages of this design, the robot operation is influenced by many factors such as: lightening, hazy climate, background lights.

In paper [4], the author designed a robot, this robot working as the following: the flex sensor sends the finger signal that connects to the Arduino Nano installed on the glove, the movements of the fingers perform certain functions such as (gyro, forward or backward). The Arduino is connected to a piece of Wi-Fi to transmit signals from the glove over a network Wi-Fi for car. The car receives the signals coming from the glove piece Wi-Fi device connected with the piece Arduino Uno, which be programmed to perform the functions of control of the car.

In paper [5], author designed a new sign gesture, the system converts the sign gesture into text as well as speech, using the gloves.

In paper [6], the author showed how the exploratory procedures, can be used to recognize the position of the finger and accordingly how to command the motors with respect to an object as well as the non-object condition.

In paper [7], the author worked on the anthropomorphic multi fingered robotic.

The paper is arranged as follows: in Section II, problem statement is presented. The hardware performance of the model is assessed in Section III. Some understanding comments are stated in Section IV. 


\section{Problem Statement}

Currently, commercialize robot are mainly for safe task that is not include in any destruction crisis and may not bring harm to the robot which can be consider as not designed for being destroy. We may need to think of the dangerous task is always bring destruction and the robot may only use for once. However, currently the production cost of a robot that designed purposely for dangerous task is very expensive, thus, it is not appropriate to be considering as using for one time only. Besides that, a robot for dangerous must be designed with time sensitive manner, which means that it should be more timely precise in doing a task and it should be task specific. As a conclusion, there is very high demand in producing a robot that is low production cost and perform a dangerous task in time sensitive manner. In this paper, the objective is to design, implement and build a low cost glove robot for dangerous task. There are several sub-objectives need to accomplish in order to successfully achieve our target.

\section{HARDWARE}

\section{L298 D IC}

This is an integrated circuit for motor drive which utilized for monitor motors in autonomous robots. It works as an interface between Arduino and the motors. This chip contains a very famous dual H -bridge. The main function of the motor driver is to control two motors independently up to 2 A, each in both directions or one stepper motor.

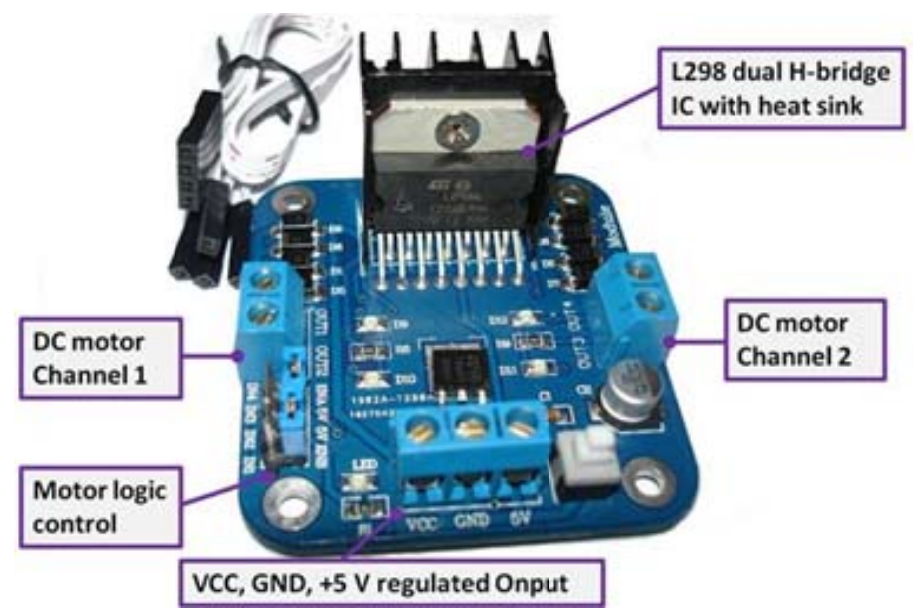

Fig. 1. L298 DIC chip

\section{Arduino Mini:}

This chip is based on a microcontroller board named AT mega 328, contains 12 digital input/ output bins, 8 analog inputs, rest button, an on board resonator, and holes for mounting pin headers.

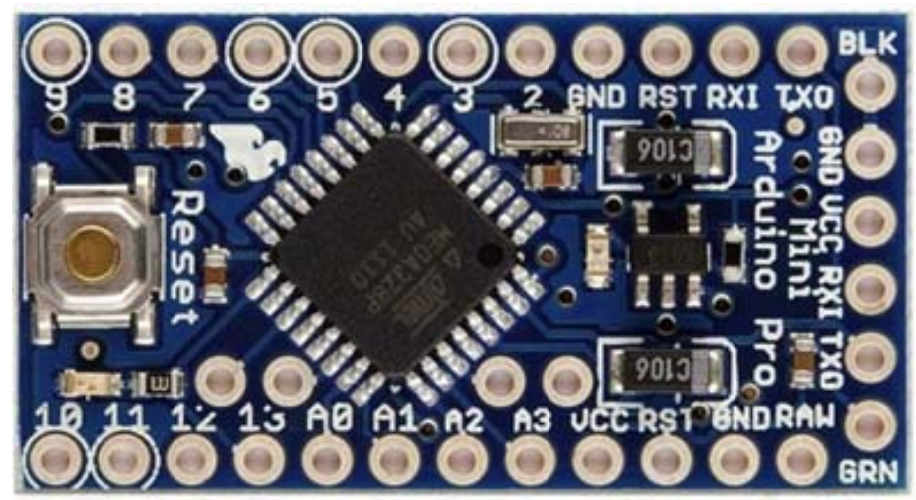

Fig. 2. Arduino Mini

Arduino Uno:

The major properties of this chip: its microcontroller broad based on the AT mega 328P, contains 14 digital input /output pins, has 6 analog inputs, it has Quartz crystal with $16 \mathrm{MHz}$ frequency. 


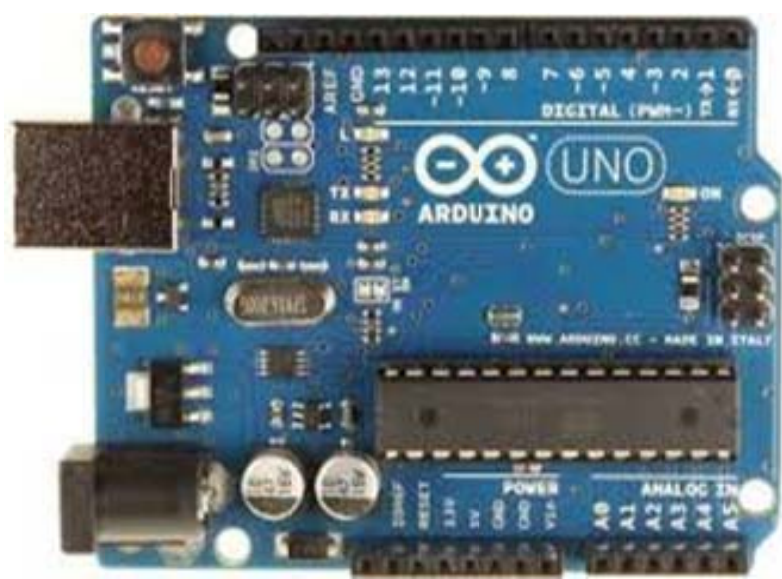

Fig. 3. Arduino Uno

\section{ESP 8266:}

The main advantage of this module it has small size and low cost which makes it proper for using as a sensor node, the specification parameters for voltage and current are: 3.3 volt, $250 \mathrm{~mA}$ respectively, it has integrated TCP/IP protocol that allow any microcontroller arrival to Wi Fi network. This module transmits commands from Arduino to L 298D to move the fans by its motors.

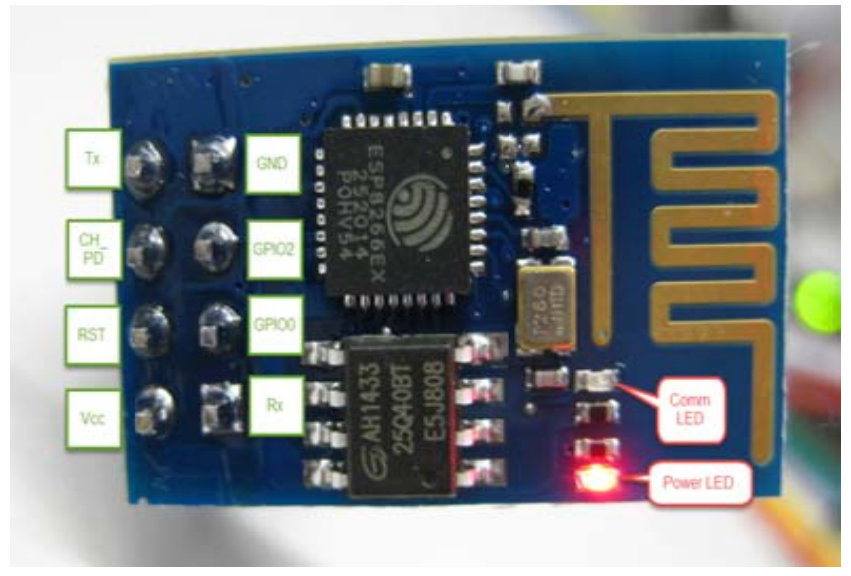

Fig. 4. ESP8266 Wi-Fi

Flex Sensor:

Flex sensor has multiple applications like most sensors. Even though it is widely used as a goniometer in rehabilitation research, its applications can be seen in different fields like, human machine interfaces, geology and musical instruments. In each application, the sensor identifies the flexure in terms of varying resistance that can be recorded digitally and the data is then used differently depending on application.

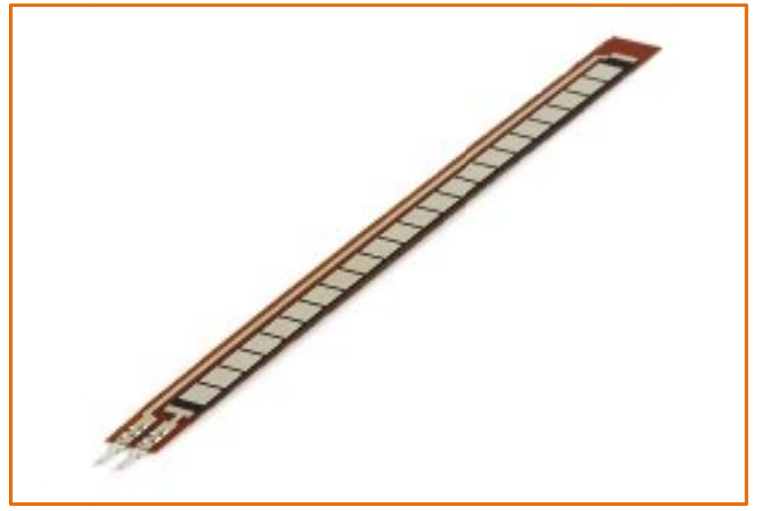

Fig. 5. Flex Sensor 
MPU 6050 Accelerometer Sensor:

MPU6050 is a nine axis motion sensor with 3 axis gyroscopes and a 3 axis accelerometer. Angle detection in industrial production and practice has a lot of applications, such as the detection of the target horizontal angle or tilt angle, can make the balance car or aircraft, to detect the target attitude, that is, the spatial angle. MPU6050 has three 16-bit ADC, to collect the acceleration value of the 3 axis or gyro value, which is converted to digital output. The range of gyroscopes measurement is plus or minus 250 degrees, plus or minus 500 degrees.

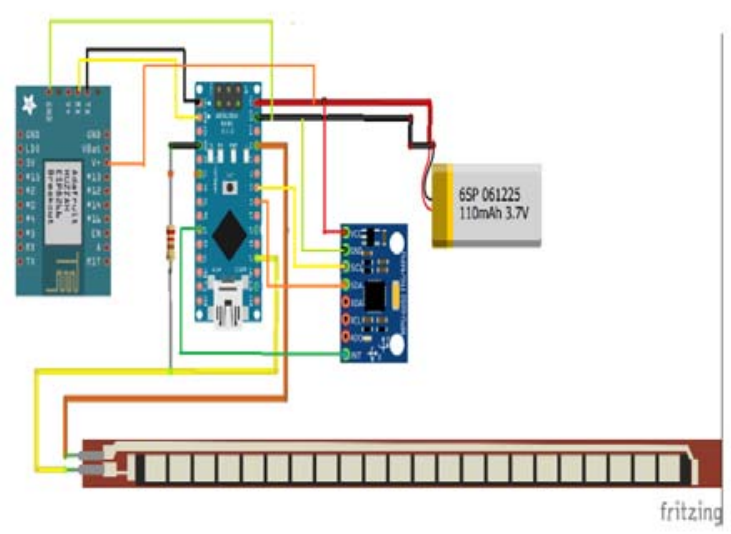

Fig. 6. Design of glove.

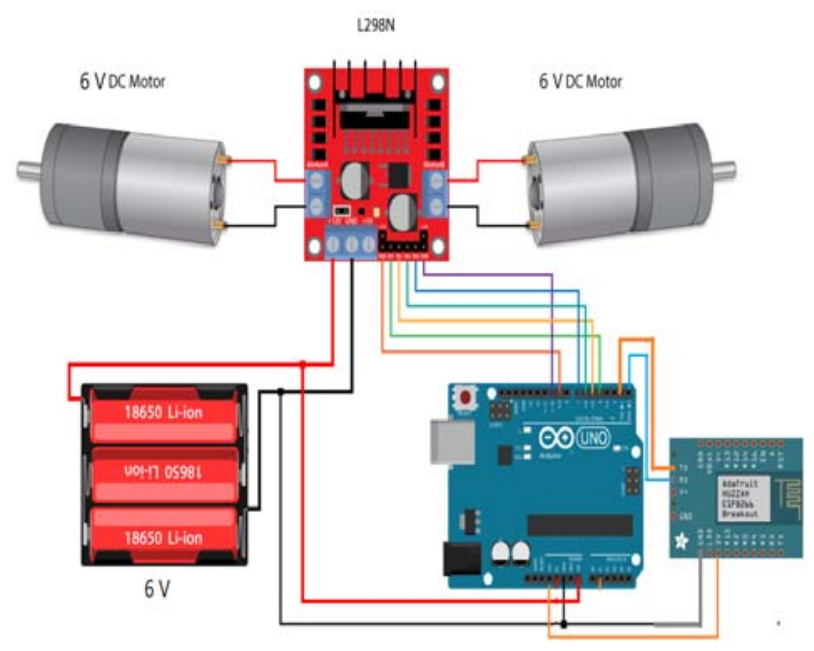

Fig. 7. Design of car.

TABLE I. Components cost

\begin{tabular}{|l|c|}
\hline \multicolumn{1}{|c|}{ Item } & Price \\
\hline Flex sensor & 18 \\
\hline Accelerometer sensor & 15 \\
\hline Arduino Nano & 15 \\
\hline Arduino Uno & 22 \\
\hline Motor drive L298n & 16 \\
\hline Motors ( 2 ) & 15 \\
\hline Connectors ( 10 ) & 10 \\
\hline Battery (3 ) & 8 \\
\hline Battery base & 2 \\
\hline glove & 2 \\
\hline Wi-Fi & $\mathbf{1 5}$ \\
\hline Total costs for project & $\mathbf{1 3 8}$ \\
\hline
\end{tabular}




\section{Overshoot}

Resistive in flex sensors show a phenomenon known as overshot: the signal peaks for a fraction of seconds, when sensor is bent fast, which occurs quite frequently as finger can go through open close cycles multiple times per second. In Figure 8, we can observe a typical overshoot pattern, in this paper, the overshoot is mitigated by choosing a minimum number of flex sensors and the best kind of the flex sensor.

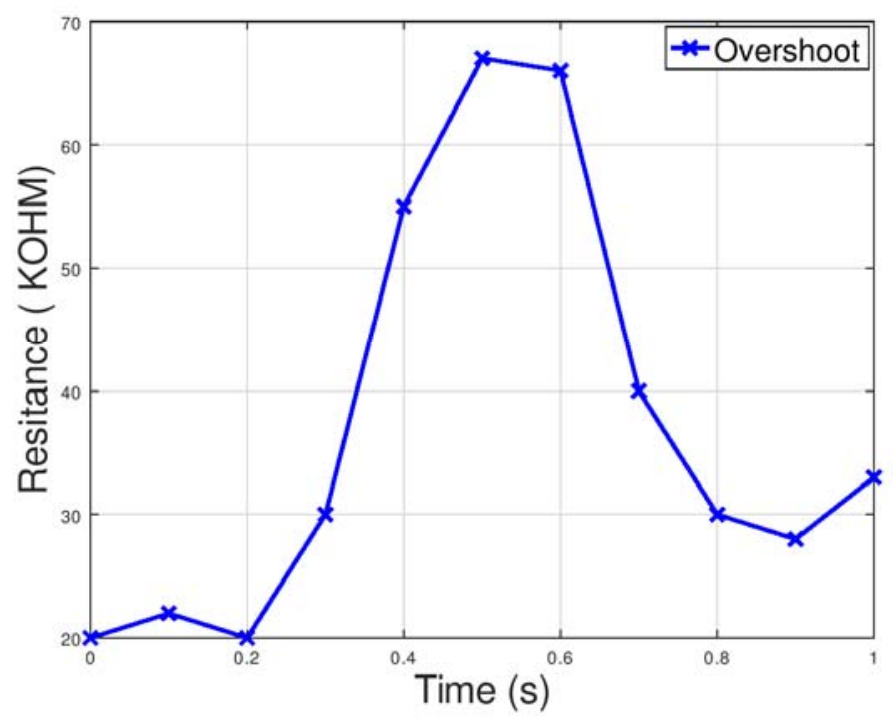

Fig. 8. The overshot response for the flex se

\section{CONCLUSION}

In this paper, we design a new magic glove to control robot with Wi -Fi controller, the main objectives of this project is to make a good contact between machine and human by controlling system with available cost for the user. Using a powerful Arduino microcontroller can handle more data. The size of robot is compact and portable.

Also we using a lighter battery to make the robot much less weight. The problems that we faced. Firstly: the values of flex sensor change rabidly that makes us to change the flex sensor more than once. Secondly: the current needed for motors is very high that made us to put motor drive to protect the motors. Finally: The need to switch batteries during a small period due to high consumption of the piece of Wi-Fi battery.

Lastly, in future we will develop this project with the following suggestions: increasing the speeds by using different gesture movements, adding more batteries on car body to make more long service, and adding more attachment like (camera, sensors) and connected to phone.

\section{AKNOWLEDEGEMENT}

We would say to thank for those support our research. We sincerely thank the anonymous reviewers for their constructive comments and suggestions. I never forget, my deep thank for my university: Palestine Technical University Kadoorei (PTUK).

\section{REFERNCES}

[1] Song M., Kim, Y., Kim, Y, and Kim, S, “A design of real time control robot system using android Smartphone”, the 7th International Conference on Ubiquitous Robots and Ambient Intelligence (URAI), Nov. 2010.

[2] S. Waldherr, R. Romero and S. Thrun, “A gesture based interface for human-robot interaction”, In Autonomous Robots, vol. 9, Issue 2, pp. 151-173, 2000.

[3] B. Wang, T. Yuan, “Traffic Police Gesture Recognition using Accelerometer”, IEEE SENSORS Conference, Lecce-Italy, pp. 10801083, Oct. 2008.Ouchi, K, “Recent trend and advance of synthetic aperture radar with selected topics,” RemoteSens. 2013 , 5, 716-807.

[4] Solanki Krunal, "Indian Sign Languages using Flex Sensor Glove” International Journal of Engineering Trends and Technology(IJEET), Vol. 4, Issue 6, 2013.

[5] K. Purohit, K. Patidar, R. Kushwah, “A Wearable Hand Gloves Gesture Detection based on Flex Sensors for disabled People”, International Journal for Research in Applied Science \& Engineering Technology (IJRASET), Vol. 5, Issues 8, 2017.

[6] A. Taksale1, A. Dangale1, I. Momin, S. Sukode, "Low Cost Robotic Hand Gloves", Journal of Electronics and Communication Engineering (IOSR-JECE), Vol. 9, Issue 2, pp. 104- 144, 2014.

[7] Cirpriani, Dario, “Closed -loop Controller for a Bio - inspired Multi -fingered under Actual Prosthesis”, Proc. of the 2006 IEEE Int. Conf. on Robotics and Automation,Orland, USA, pp. 2111- 2116. 


\section{AUTHOR}

RAED S. M. DARAGHMA was born in 1977 in Palestine, he received his master degree from Jordan science and technology in electrical and communication engineering Jordan in 2010, he got his P.H.D degree from anadolu university, turkey in 2016. He has published number of papers and journals. He has engaged in educational work many years manly teaches digital communication, mobile, digital communication networks at Palestine technical university P.T.U. His mainly research areas include wireless sensor networks, signal processing and MIMO radar. 